International Journal of Mathematics Research.

ISSN 0976-5840 Volume 12, Number 1 (2020), pp. 33-37

(C) International Research Publication House

https://dx.doi.org/10.37624/IJMR/12.1.2020.33-37

\title{
A Subclass of Univalent Functions Defined by Ruscheweyh Operator
}

\author{
Timilehin G. Shaba \\ Department of Mathematics, University of Ilorin, Ilorin, Nigeria.
}

\begin{abstract}
The main object of this paper is to introduce a new class $\mathcal{G J}_{n}(m, \lambda, \alpha)$ define by Ruscheweyh operator involving function $f(z) \in \mathcal{A}_{n}$. Parallel results, for some related classes including the class of starlike, convex and Bazilevic functions respectively, are also obtained.
\end{abstract}

Keywords: Univalent function, starlike function, convex function, Bazilevic function, Ruscheweyh Operator.

\section{INTRODUCTION AND DEFINITIONS}

Let $\mathcal{A}_{n}$ denote the class of function of the form

$$
f(z)=z+\sum_{k=n+1}^{\infty} a_{k} z^{k}
$$

which are analytic in the open unit disc $\mathbb{U}=\{z \in \mathbb{C}:|z|<1\}$ and the space of holomorphic functions in $\mathbb{U}, n \in \mathbb{N}=\{1,2,3, \cdots\}$.

Let $\mathcal{J}_{n}$ denote the subclass of functions that are univalent in $\mathbb{U}$.

By $S_{n}^{*}(\alpha) \subset \mathcal{J}_{n}$ denote the subclass of starlike functions of order $\alpha, 0 \leq \alpha<1$ which satisfies the condition

$$
\Re\left(\frac{z f^{\prime}(z)}{f(z)}\right)>\alpha \quad(z \in \mathbb{U}) .
$$

Further, a function $f(z)$ belonging to $\mathcal{K}_{n}(\alpha) \subset \mathcal{J}_{n}$ is said to be convex functions of order $\alpha$, $0 \leq \alpha<1$ in $\mathbb{U}$, if and only if

$$
\Re\left(\frac{z f^{\prime \prime}(z)}{f^{\prime}(z)}+1\right)>\alpha \quad(z \in \mathbb{U}),
$$


and denote by $\mathcal{R}_{n}(\alpha)$ the class of functions in $\mathcal{J}_{n}$ which satisfy the condition

$$
\Re\left\{f^{\prime}(z)\right\}>\alpha \quad(z \in \mathbb{U}) .
$$

It is well known that $\mathcal{K}_{n}(\alpha) \subset S_{n}^{*}(\alpha) \subset \mathcal{J}_{n}$.

In [5] Ruscheweyh has define the operator

$$
\begin{gathered}
D^{h}: \mathcal{A}_{n} \longrightarrow \mathcal{A}_{n}, n \in \mathbb{N}=\{1,2,3,4, \cdots\}, \\
D^{0} f(z)=f(z) \\
D^{1} f(z)=z f^{\prime}(z) \\
(h+1) D^{h+1} f(z)=z\left[D^{h} f(z)\right]^{\prime}+h D^{h} f(z) \quad(z \in \mathbb{U}),
\end{gathered}
$$

We note that if $f \in \mathcal{A}_{n}$, then

$$
D^{h} f(z)=z+\sum_{k=n+1}^{\infty} \frac{(h+k-1) !}{h !(k-1) !} a_{k} z^{k} \quad(z \in \mathbb{U}) .
$$

where $h \in \mathbb{N} \cup\{0\}=\{0,1,2,3, \ldots\}$.

To prove our main theorem we shall need the following lemma.

Lemma 1.1. [3] Let $q$ be analytic in $\mathbb{U}$ with $q(0)=1$ and suppose that

$$
\Re\left(1+\frac{z q^{\prime}(z)}{q(z)}\right)>\frac{3 \alpha-1}{2 \alpha} \quad(z \in \mathbb{U}) .
$$

Then $\Re(q(z))>\alpha$ in $\mathbb{U}$ and $\frac{1}{2} \leq \alpha<1$.

\section{MAIN RESULTS}

Definition 2.1. A function $f(z) \in \mathcal{A}_{n}$ is said to be a member of the class $\mathcal{G J}_{n}(h, \lambda, \alpha)$ if

$$
\left|\frac{D^{h+1} f(z)}{z}\left(\frac{D^{h} f(z)}{z}\right)^{\lambda}-1\right|<1-\alpha, \quad z \in U \quad, \quad \lambda \geq-2 \quad \text { and } \quad \frac{1}{2} \leq \alpha<1
$$

where $D^{h}$ is the Ruscheweyh operator. Note that inequality (2.1) implies that

$$
\operatorname{Re}\left(\frac{D^{h+1} f(z)}{z}\left(\frac{D^{h} f(z)}{z}\right)^{\lambda}\right)>\alpha . \quad \frac{1}{2} \leq \alpha<1 .
$$


Remark 2.2. The function family $\mathcal{G J}_{n}(h, \lambda, \alpha)$ is a comprehensive class of analytic functions which includes some known and new classes of analytic and univalent functions. For example,

1. $\mathcal{G J}_{n}(0,-1, \alpha) \equiv S_{n}^{*}(\alpha)$,

2. $\mathcal{G J}_{n}(1,-1, \alpha) \equiv \mathcal{K}_{n}(\alpha)$,

3. $\mathcal{G J ~}_{n}(0,0, \alpha) \equiv \mathcal{R}_{n}(\alpha)$,

4. $\mathcal{G J}_{1}(0, \lambda, \alpha) \equiv \mathcal{B}(\lambda, \alpha)=$

$\left\{f \in \mathcal{A}:\left|f^{\prime}(z)\left(\frac{f(z)}{z}\right)^{\lambda}-1\right|<1-\alpha ; \lambda \geq-1,0 \leq \alpha<1, z \in \mathbb{U}\right\}$

intoduced by Singh [4] and studied by Babalola[1].

5. $\mathcal{G S}_{1}(0,-2, \alpha) \equiv \mathcal{B}(\alpha)$ studied by Frasin and Darus $[2]$.

Theorem 2.3. If $f(z) \in \mathcal{A}_{n}$ satisfies the condition

$$
\Re\left(\frac{(h+2) D^{h+2} f(z)}{D^{h+1} f(z)}+\frac{\lambda(h+1) D^{h+1} f(z)}{D^{h} f(z)}-(1+\lambda)(h+1)\right)>\frac{3 \alpha-1}{2 \alpha}
$$

then $f(z) \in \mathcal{G}_{n}(h, \lambda, \alpha)$

Proof. For $z \in \mathbb{U}$, define an analytic function $q(z)$ with $q(0)=1$ by

$$
q(z)=\frac{D^{n+1} f(z)}{z}\left(\frac{D^{n} f(z)}{z}\right)^{\lambda}
$$

By simplification,

$$
\ln q(z)=\ln \left(D^{h+1} f(z)\right)-\ln (z)+\lambda \ln \left(D^{h} f(z)\right)-\lambda \ln (z)
$$

and by simple differentiation it implies that

$$
\frac{q^{\prime}(z)}{q(z)}=\frac{\left(D^{h+1} f(z)\right)^{\prime}}{\left(D^{h+1} f(z)\right)}-\frac{1}{z}+\lambda \frac{\left(D^{h} f(z)\right)^{\prime}}{\left(D^{h} f(z)\right)}-\lambda \frac{1}{z}
$$

so that,

$\Re\left(1+\frac{z q^{\prime}(z)}{q(z)}\right)=\Re\left(\frac{(h+2) D^{h+2} f(z)}{D^{h+1} f(z)}+\frac{\lambda(h+1) D^{h+1} f(z)}{D^{h} f(z)}-(1+\lambda)(h+1)\right)>\frac{3 \alpha-1}{2 \alpha}$

which, by Lemma 1.1, implies

$$
\operatorname{Re}\left(\frac{D^{n+1} f(z)}{z}\left(\frac{D^{n} f(z)}{z}\right)^{\lambda}\right)>\alpha, \quad\left(\frac{1}{2} \leq \alpha<1\right) .
$$


Remark 2.4. When $n=1$ and $h=0$ in Theorem 2.3, we have the following theorem.

Theorem 2.5. [1] If $f \in \mathcal{A}$ satisfies

$$
\Re\left\{\left(1+\frac{z f^{\prime \prime}(z)}{f^{\prime}(z)}\right)+\lambda \frac{z f^{\prime}(z)}{f(z)}\right\}>\lambda+\frac{3 \alpha-1}{2 \alpha}, \quad z \in \mathbb{U}
$$

Then $\Re(q(z))>\alpha$ in $\mathbb{U}$ and $\frac{1}{2} \leq \alpha<1$.

From Theorem 2.3, the following Corollaries hold true.

Corollary 2.6. If $f(z) \in \mathcal{A}_{n}$, and

$$
\Re\left\{\frac{4 z^{2} f^{\prime \prime}(z)+z^{3} f^{\prime \prime \prime}(z)+2 z f^{\prime}(z)}{z^{2} f^{\prime \prime}(z)+2 z f^{\prime}(z)}\right\}>\frac{1}{2} \quad(z \in \mathbb{U}),
$$

then

$$
\Re\left[z f^{\prime \prime}(z)+f^{\prime}(z)\right]>\frac{1}{2}, \quad(z \in \mathbb{U}) .
$$

Corollary 2.7. If $f(z) \in \mathcal{A}_{n}$ and

$$
\Re\left\{\frac{z f^{\prime \prime}(z)}{f^{\prime}(z)}-\frac{z f^{\prime}(z)}{f(z)}\right\}>-\frac{3}{2} \quad(z \in \mathbb{U}),
$$

then

$$
\Re\left[\frac{z f^{\prime}(z)}{f(z)}\right]>\frac{1}{2} .
$$

That is $f(z)$ is starlike of order $\frac{1}{2}$, hence $f \in \mathcal{G J}_{n}\left(0,-1, \frac{1}{2}\right) \equiv S_{n}^{*}(\alpha)$.

Corollary 2.8. If $f(z) \in \mathcal{A}_{n}$ and

$$
\Re\left\{1+\frac{z f^{\prime \prime}(z)}{f^{\prime}(z)}\right\}>\frac{1}{2} \quad(z \in \mathbb{U})
$$

then

$$
\Re\left[f^{\prime}(z)\right]>\frac{1}{2} .
$$

In another words, if the function $f(z)$ is convex of order $\frac{1}{2}$, then $f(z) \in \mathcal{G J}_{n}\left(0,0, \frac{1}{2}\right) \equiv \mathcal{R}_{n}\left(\frac{1}{2}\right)$

Corollary 2.9. If $f(z) \in \mathcal{A}_{n}$ and

$$
\Re\left\{2\left(\frac{z f^{\prime \prime}(z)}{f^{\prime}(z)}+1\right)-\frac{z f^{\prime}(z)}{f(z)}\right\}>0 \quad(z \in \mathbb{U}),
$$

then

$$
\Re\left[\frac{z^{\frac{1}{2}} f^{\prime}(z)}{f^{\frac{1}{2}}(z)}\right]>\frac{1}{2} .
$$

That is $f(z)$ is Bazilevic of order $\frac{1}{2}$, type $\frac{1}{2}$ in $\mathbb{U}$. 
Corollary 2.10. If $f(z) \in \mathcal{A}_{n}$ and

$$
\Re\left\{2\left(\frac{z f^{\prime \prime}(z)}{f^{\prime}(z)}+1\right)+\frac{z f^{\prime}(z)}{f(z)}\right\}>1 \quad(z \in \mathbb{U}),
$$

then

$$
\Re\left[\frac{f^{\frac{1}{2}}(z) f^{\prime}(z)}{z^{\frac{1}{2}}}\right]>\frac{1}{2} .
$$

That is $f(z)$ is Bazilevic of order $\frac{1}{2}$, type $\frac{3}{2}$ in $\mathbb{U}$.

\section{CONCLUSION}

In this paper, using Ruscheweyh operator, we defined new subclass of univalent function and established some of its properties. Results obtained provide properties of certain subclasses of univalent functions.

\section{REFERENCES}

[1] K.O. Babalola, 2014," Combinations of geometric expressions implying schlichtness," Analele Universitatii din Oradea, vol. 1, pp. 91-94.

[2] B.A. Frasin and M. Darus, 2001,'On certain analytic univalent functions," International Journal of Mathematics and Mathematical Sciences , vol. 25, pp. 305-310.

[3] B.A. Frasin and J.M. Jahangiri, 2008,"A new and comprehensive class of analytic functions," Analele Universitatii din Oradea, vol. 15, pp. 61-64.

[4] R. Singh, 1973, "On bazilevic functions," Proceedings of the American Mathematical Society, vol. 38, pp. 261-271.

[5] St. Ruscheweyh, 1975, "New criteria for univalent functions," Proceedings of the American Mathematical Society, vol. 49, pp. 109-115. 